\title{
Resveratrol alleviates diabetic mechanical allodynia in rats by downregulating P2X3R
}

\author{
YUANYUAN CUI ${ }^{1}$, YUTING LI ${ }^{2}$, JIAYI NING ${ }^{2}$, YAJING MI ${ }^{1}$, XIAOLONG WANG ${ }^{1}$, \\ ZHONGYING QIU ${ }^{1}$, LE LI $^{1}$ and XINGCHUN GOU ${ }^{1}$ \\ ${ }^{1}$ School of Basic Medical Sciences and Shaanxi Key Laboratory of Brain Disorders, \\ ${ }^{2}$ School of Clinical Medicine, Xi'an Medical University, Xi'an, Shaanxi 710021, P.R. China
}

Received July 3, 2019; Accepted April 17, 2020

DOI: $10.3892 / \mathrm{mmr} .2020 .11157$

\begin{abstract}
Mechanical allodynia, which develops in patients of diabetes mellitus as a neuropathic manifestation, remains without an effective treatment. The aim of the present study was to investigate the effects and potential mechanisms underlying resveratrol (RES) in a rat model of streptozocin (STZ)-induced diabetic mechanical allodynia (DMA). The rat model of DMA was established by the administration of an intraperitoneal injection of STZ. From day 8 post-STZ injection, rats were administered with an intragastric injection of various doses of RES for 14 consecutive days. The von Frey filaments were applied to detect the paw withdrawal threshold and evaluate the analgesic effects of RES. Based on the dose-effect curve, the $\mathrm{ED}_{50}$ of RES was calculated. Immunofluorescence staining and western blotting were performed to detect the expression of purinergic receptor P2X3 (P2X3R) in the dorsal root ganglion (DRG) and spinal dorsal horn (SDH) following $\mathrm{RES}_{\mathrm{ED} 50}$ treatment. The results indicated that RES significantly alleviated mechanical allodynia in DMA model rats in a dose-dependent manner. Compared with the control group, the expression of P2X3R in DRG neurons and SDH terminals was markedly decreased following the administration of $\mathrm{RES}_{\mathrm{ED} 50}(\mathrm{P}<0.05)$. Collectively, the results indicated that RES displayed a dose-dependent analgesic effect on DMA model rats. Furthermore, P2X3R expression downregulation in the DRG and SDH may be a mechanism underlying the analgesic effects of RES on DMA-related behaviors.
\end{abstract}

Correspondence to: Professor Xingchun Gou or Professor Le Li, School of Basic Medical Sciences and Shaanxi Key Laboratory of Brain Disorders, Xi'an Medical University, 1 Xinwang Road, Weiyang, Xi'an, Shaanxi 710021, P.R. China

E-mail: gouxingchun@189.cn

E-mail: 1428529763@qq.com

Key words: resveratrol, diabetes mellitus, mechanical allodynia, purinergic receptor $\mathrm{P} 2 \mathrm{X} 3$

\section{Introduction}

Diabetes mellitus (DM) is a metabolic disease that is primarily characterized by hyperglycemia (1). Diabetic neuropathic pain (DNP) is one of the intractable complications of DM and $\sim 30 \%$ of patients with diabetes suffer from DNP (2), which can result in peripheral nerve dysfunctions, such as nerve degeneration, allodynia, hyperalgesia and insensitivity (3). Thermal hyperalgesia and mechanical allodynia are the most common forms of DNP (4). Compared with thermal hyperalgesia, diabetic mechanical allodynia (DMA) severely affects the quality of life of patients with diabetes (5). To a certain extent, thermal hyperalgesia can be avoided by reducing contact with heat sources; however, mechanical stimulation is ubiquitous and can cause mechanical allodynia in patients when they are performing everyday tasks, such as getting dressed (5). The molecular mechanisms underlying DMA development and maintenance are not completely understood; therefore, the clinical prevention and treatment of DMA is a challenge.

Resveratrol $(3,4$ ',5,-trihydroxystilbene; RES) is a natural polyphenolic phytoalexin with a number of beneficial abilities, such as neuroprotective, antitumorigenic, antioxidative stress and anti-inflammatory effects (6-8). RES promotes nerve regeneration, and improves the pathological and behavioral outcomes of various peripheral and central nerve injuries, including sciatic nerve crush (9), nerve root avulsion (10), chronic constriction of dorsal root ganglion (DRG) (11), traumatic brain (12) and spinal cord (13) injuries. RES can also alleviate pain by decreasing inflammatory responses via inhibition of proinflammatory cytokines and induction of anti-inflammatory cytokine release in the nerves or local tissue (14). In addition, the analgesic effect of RES on trigeminal neuralgia involves suppression of glial activation (15). It has been reported that RES alone or in combination with insulin or 4-amino 1,8 naphthalimide (4-ANI) can attenuate thermal hyperalgesia from the 4-8th week following the administration of streptozocin (STZ) in rats (16-18). However, whether RES influences mechanical allodynia within the 4 weeks following the injection of STZ is still not completely understood.

ATP is a major signaling molecule in cell communication and a major neurotransmitter in the nervous system (19). The purinergic receptor $\mathrm{P} 2 \mathrm{X} 3$ ( $\mathrm{P} 2 \mathrm{X} 3 \mathrm{R})$ can be activated by ATP release following peripheral tissue damage. $\mathrm{P} 2 \mathrm{X} 3 \mathrm{R}$ is 
primarily localized on small nociceptive sensory neurons in the DRG, trigeminal ganglia and nodose ganglia, and serves an important role in nociceptive transmission (20). The expression and function of P2X3R are markedly enhanced in DRG neurons in diabetic model rats, and hindpaw pain hypersensitivity is attenuated by the injection of a P2X3R antagonist (21). Recently, it was reported that RES can alleviate neuropathic pain in a model of partial sciatic nerve ligation ( $\mathrm{pSNL}$ ) by suppressing increases in P2X3R and ERK phosphorylation in the DRG (22). Furthermore, it has been reported that protein expression levels of P2X3R in DRG neurons and spinal dorsal horn (SDH) fibers are increased from the 2nd-4th week in a rat model of STZ-induced DM (23). However, whether the analgesic role of RES is associated with $\mathrm{P} 2 \mathrm{X} 3 \mathrm{R}$ expression in DMA is not completely understood.

Therefore, the present study investigated the effects of RES on DMA-related behaviors in rats. Moreover, to investigate the analgesic role and possible mechanisms underlying RES, alterations to the expression of P2X3R in the DRG and SDH following $\operatorname{Res}_{\mathrm{ED} 50}$ treatment were assessed.

\section{Materials and methods}

Animal preparation. Male Sprague-Dawley rats (weight, 180-220 g; age, 6-7 weeks) were supplied by the Experimental Animal Center of Xi'an Jiao Tong University. All rats were housed in an environment with a controlled temperature $\left(22-25^{\circ} \mathrm{C}\right)$ and humidity (50-65\%) with 12 -h light/dark cycles, and free access to food and water. Rats were allowed to adapt to the environment for 7 days prior to subsequent experiments. The present study was approved by the Animal Care and Ethical Committee at Xi'an Medical University and all animal experiments were performed according to the Guidelines of the International Association for the Study of Pain (24). All efforts were made to minimize animal suffering and to follow the 3Rs (reduction, refinement and replacement).

Preparation of the rat model of DMA: Experiment (Exp) 1. The preparation of the rat model of DMA is presented in Fig. 1. Initially, a rat model of DM was established. STZ (Sigma-Aldrich; Merck KGaA) was diluted using citrate buffer (v:v=1:1.32; pH 4.5; cat. no. C1949; Tokyo Chemical Industry UK Ltd.). The rats were randomly divided into two groups: i) The DM group received an intraperitoneal injection of $60 \mathrm{mg} / \mathrm{kg} \mathrm{STZ}$; and ii) the vehicle group received an equal volume of citrate buffer.

On 1 day prior to and 7, 14, 21 and 28 days post-STZ injection, blood glucose, body weight and paw withdrawal threshold (PWT) were measured. Rats with a non-fasting blood glucose concentration $>16.7 \mathrm{mmol} / \mathrm{l}(300 \mathrm{mg} / \mathrm{dl})$ at $72 \mathrm{~h}$ after the STZ injection were considered as DM model rats. Body weight was also considered as an additional index for successful establishment of the rat model of DM. Compared with the vehicle group, the increased body weight in DM model rats was slow, and there was a significant difference between two groups $(\mathrm{P}<0.01)$. Mechanical allodynia was detected using flexile von Frey filaments to select for DMA model rats among DM model rats, as previously described (25). Briefly, the rats were individually placed in Plexiglas ${ }^{\circledR}$ boxes for $\sim 20 \mathrm{~min}$ to allow them to acclimate to the testing environment prior to the experiment. Subsequently, von Frey filaments (0.6-15 g; Stoelting Co.) were pressed perpendicularly on the plantar surface of the hind paw and were bent with sufficient force for $\sim 5$ sec. Each test was repeated at least 5 times at 3-min intervals. The PWT was recorded as the minimal value resulting in at least 3 incidences of brisk withdrawal or paw flinching. PWT tests were performed on 1 day prior to and 7, 14, 21 and 28 days post-STZ administration to estimate DMA-related pain behaviors. Rats displaying an obvious decline in PWT on day 7 post-STZ injection were regarded as DMA model rats. All behavioral experiments were blinded and were performed between 09:00-18:00. Based on the results of PWT on the 7 th day after STZ injection, a total of 99 DMA model rats were randomly divided into groups: i) $\operatorname{Exp} 1(n=15)$; ii) $\operatorname{Exp} 2(n=60)$; and iii) $\operatorname{Exp} 3(n=24)$. A further 27 vehicle rats were used in the present study (15 in Exp 1 and 12 in Exp 3). A total of 220 rats were used in the present study, among which 126 (DMA 99 and vehicle 27) were used as effective experimental rats for the Exp1-3 and including 94 rats that were not successfully prepared as DMA model rats by STZ administration.

Evaluation of the analgesic effect of RES and calculation of the $R E S_{E D 50}$. The aim of Exp 2 was to evaluate the repetitive intragastric administration of various doses of RES on DMA-related behaviors and to calculate the $\operatorname{RES}_{\mathrm{ED} 50}$ (Fig. 1). RES (Sigma-Aldrich; Merck KGaA) was diluted using alcohol-saline (AS; v:v=1:5). DMA model rats were randomly divided into the following four groups ( $n=15$ per group): i) $\mathrm{DMA}+\mathrm{AS}$; ii) DMA + RES (25 mg/kg); iii) DMA + RES (100 mg/kg); and iv) DMA + Res (400 mg/kg). DMA + AS group rats received the same volume of AS and were regarded as the control groups. The doses of RES selected for the present study were based on a previous report (15). All groups received AS/RES for 14 consecutive days from day 8-21 post-STZ injection.

PWTs were measured weekly until one week post-RES/AS withdrawal. The area under the time-course curve (AUC) values were used to evaluate the overall effect of RES, according to previous studies (26-30). The goodness of fit was presented as the followings: Degrees of freedom $=56 ; \mathrm{R}^{2}=0.8025$; absolute sum of squares $=11454$; and $S y \cdot x=60$. The $E_{50}$ was calculated from the best fitting curve. The doses of intragastrically administered RES were transformed into the logarithm dose and the non-line fit was created to establish the dose-effect curve. Based on the dose-effect curve, $\operatorname{RES}_{\mathrm{ED} 50}(\mathrm{ED} 50=$ median effective dose) was calculated according to the following formula: $Y=E m i n+(E m a x-E m i n) /\left[1+10^{\wedge}((\operatorname{LogED} 50-X) x\right.$ HillSlope)]. Best-fit values in the formula were: Emin $=4.359 \%$, Emax $=70.460 \%$, HillSlope $=1.675, Y=50 \%$, Best-fit $X=2.170$.

Assessment of alterations in the expression of $P 2 X 3 R$ after the administration of $R E S_{E D 50}$. The effect of $\mathrm{RES}_{\mathrm{ED} 50}$ on $\mathrm{P} 2 \mathrm{X} 3 \mathrm{R}$ protein expression in the DRG and SDH was assessed (Exp 3; Fig. 1). The 24 DMA rats and 12 vehicle rats were equally used for immunofluorescence staining and western blotting, respectively. Then for each approach, rats were randomly divided into three groups as follows ( $n=6$ per group): i) The vehicle + AS group, which consisted of vehicle rats receiving AS; ii) the DMA + AS group, which consisted of DMA model rats receiving $\mathrm{AS}$; and iii) the $\mathrm{DMA}+\mathrm{RES}_{\mathrm{ED} 50}$ group, which 

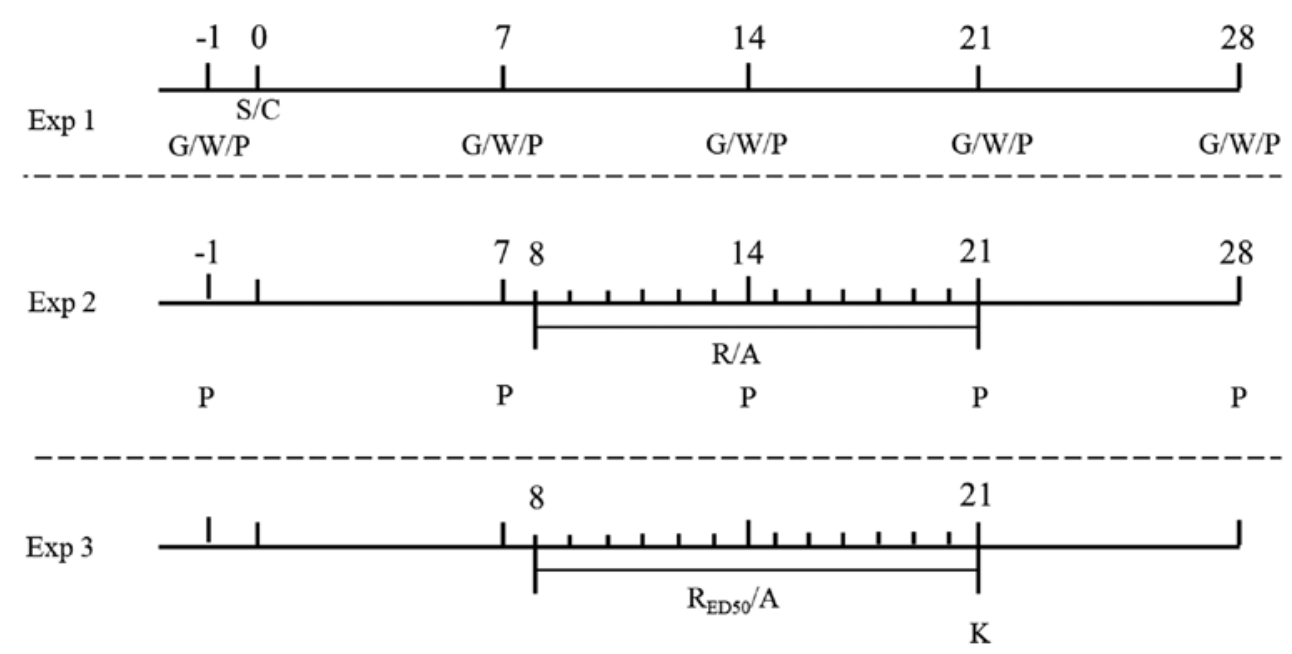

Figure 1. Experimental protocols. S, streptozocin; C, citrate buffer; G, blood glucose; W, body weight; P, paw withdrawal threshold; R, resveratrol; A, alcohol-saline; $\mathrm{K}$, euthanization of rats; exp, experiment.

consisted of DMA model rats receiving $\mathrm{RES}_{\mathrm{ED} 50}$. Rats received $\mathrm{AS} / \mathrm{RES}_{\mathrm{ED} 50}$ for 14 consecutive days from day 8-21 post-STZ injection.

Immunofluorescence staining. Following 14 days of continuous administration of $\mathrm{AS} / \mathrm{RES}_{\mathrm{ED} 50}$, rats were anaesthetized by the intraperitoneal injection of sodium pentobarbital $(65 \mathrm{mg} / \mathrm{kg})$. Subsequently, rats were transcardially perfused with $100 \mathrm{ml}$ $0.9 \%$ normal saline, followed by $500 \mathrm{ml} \mathrm{PBS} \mathrm{(pH} \mathrm{7.2;} 0.1 \mathrm{~mol} / \mathrm{l}$ ) containing $4 \%$ paraformaldehyde. The $\mathrm{L}_{4-5}$ spinal segments and the corresponding DRGs were harvested and post-fixed in $4 \%$ paraformaldehyde for $2-4 \mathrm{~h}$ at $4^{\circ} \mathrm{C}$. Subsequently, the tissues were cryoprotected overnight at $4^{\circ} \mathrm{C}$ in $0.1 \mathrm{~mol} / 1 \mathrm{PBS}$ containing $30 \%$ sucrose. Frozen transverse sections of spinal cord (SC) (thickness, $30 \mu \mathrm{m}$ ) and horizontal sections of DRG (thickness, $10 \mu \mathrm{m}$ ) were cut using a CM1800 cryostat (Leica Microsystems $\mathrm{GmbH}$ ). The sections were immunofluorescently stained to detect P2X3R expression. Briefly, tissue sections were rinsed with $0.01 \mathrm{~mol} / 1 \mathrm{PBS}$ (pH 7.2) and blocked with 10\% FBS (cat. no. F8687; Sigma-Aldrich; Merck KGaA) in PBS $(0.01 \mathrm{~mol} / \mathrm{l})$ for $1 \mathrm{~h}$ at room temperature. Subsequently, the sections were incubated with a rabbit anti-P2X3R antibody (cat. no. ab10269; 1:1,000; Abcam) at $4^{\circ} \mathrm{C}$ overnight. Following primary incubation, the sections were incubated with a biotinylated donkey anti-rabbit IgG (cat. no. AP182F; 1:500; EMD Millipore) secondary antibody at room temperature for 6-8 h. The sections were also incubated with FITC-labeled avidin D (cat. no. A-2001; 1:1,000; Vector Laboratories, Inc.) with DAPI (cat. no. D9542; 1:1,000; Sigma-Aldrich; Merck KGaA) at room temperature for $2 \mathrm{~h}$. DAPI staining was performed to count the number of DRG neurons (data not shown). Between each step, the sections were rinsed with PBS (0.01 mol/l). The incubation medium used for the primary and second antibodies was PBS $(0.01 \mathrm{~mol} / \mathrm{l} ; \mathrm{pH}$ 7.4) supplemented with $2 \%$ normal donkey serum (cat. no. ZB-2301; OriGene Technologies, Inc.), $0.3 \%$ Triton $\mathrm{X}-100$ (pH 7.4), $0.25 \% \lambda$-carrageen (cat. no. C1013; Sigma-Aldrich; Merck KGaA) and $0.05 \%$ sodium azide. The incubation medium used for FITC-avidin was PBS (0.01 mol/1; pH 7.4) supplemented with $0.3 \%$ Triton X-100. Stained sections were observed using a FV1000 confocal laser scanning microscope (Olympus Corporation; magnification, $x 100)$. The optical density of P2X3R-immunoreactivity in DRG or spinal dorsal horn (SDH) was measured by ImageJ (v1.46r; National Institutes of Health) and the percentage of P2X3R positive cells over total neurons were calculated.

Western blotting. Rats were anesthetized by the intraperitoneal injection of sodium pentobarbital, and the $\mathrm{L}_{4-5}$ segments of SDH and bilateral DRGs were immediately harvested on ice, as previously described (31). The protein was extracted from SDHs and DRGs using the lysate (RIPA:PMSF=100:1; RAPI; cat. no. P0013; Beyotime Institute of Biotechnology; PMSF; cat. no. ST506; Beyotime Institute of Biotechnology). The protein concentration of samples was measured by the bicinchoninic acid method using the albumin standard (cat. no. 23209; Thermo Fisher Scientific, Inc.). Briefly, after heating at $100^{\circ} \mathrm{C}$ for $5 \mathrm{~min}, 30 \mu \mathrm{g}$ of protein were separated via SDS-PAGE on a $10 \%$ gel and transferred onto PVDF membranes (EMD Millipore). The membranes were blocked with TBS containing $0.02 \%$ Tween (TBST) and 5\% non-fat dry milk for $1 \mathrm{~h}$ at room temperature. Subsequently, the membranes were incubated overnight at $4^{\circ} \mathrm{C}$ with the following primary antibodies: Anti-P2X3R (rabbit; cat. no. ab10269; $1: 1,000$; Abcam) or anti- $\beta$-actin (mouse; cat. no. A228; 1:5,000; Sigma-Aldrich; Merck KGaA). Following primary incubation, the membranes were incubated with horseradish peroxidase-conjugated anti-rabbit (cat. no. AP307P; 1:5,000; EMD Millipore) or anti-mouse (cat. no. AP124P; 1:5,000; EMD Millipore) secondary antibodies for $1 \mathrm{~h}$ at room temperature. Between each step, the membranes were rinsed three times with TBST for 10 min each. Proteins were visualized with the ECL kit (cat. no. 32132; Thermo Fisher Scientific, Inc.). Protein expression levels were quantified using the ChemiDoc ${ }^{\mathrm{TM}} \mathrm{MP}$ Imaging System (Bio-Rad Laboratories, Inc.) with $\beta$-actin as the loading control.

Statistical analysis. Data are presented as the mean \pm SEM. Researchers were blinded to the behavioral test and reagents used. The data of Figs. 2 and 3A were analyzed by repeated measures ANOVA, and Fig. 4G-I were analyzed using one-way 

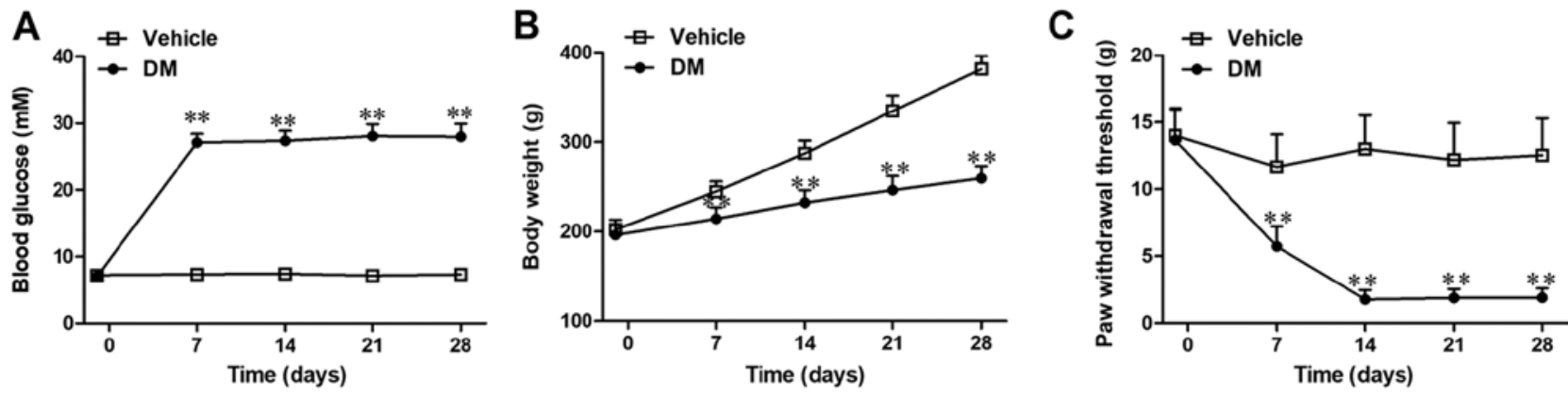

Figure 2. STZ-induced rat models of DM and DMA. Alterations to (A) blood glucose concentration, (B) body weight and (C) paw withdrawal threshold after administration of STZ or vehicle (citrate buffer). The results were repeatedly measured 5 times on 1 day pre- and 7, 14, 21 and 28 day post-STZ injections, respectively. ${ }^{* *} \mathrm{P}<0.01$ vs. vehicle. STZ, streptozocin; DM, diabetes mellitus; DMA, diabetic mechanical allodynia.

A

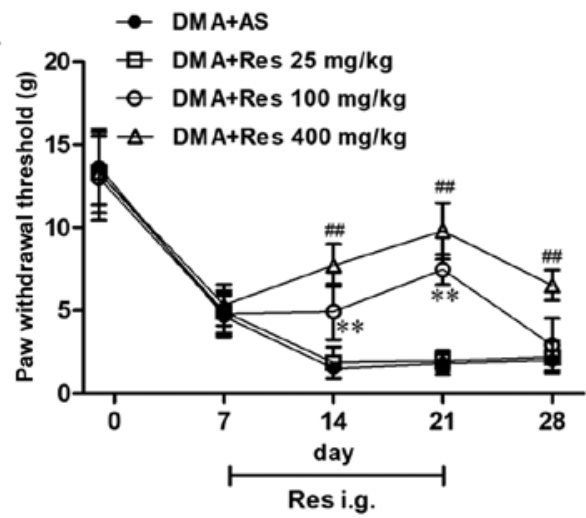

C

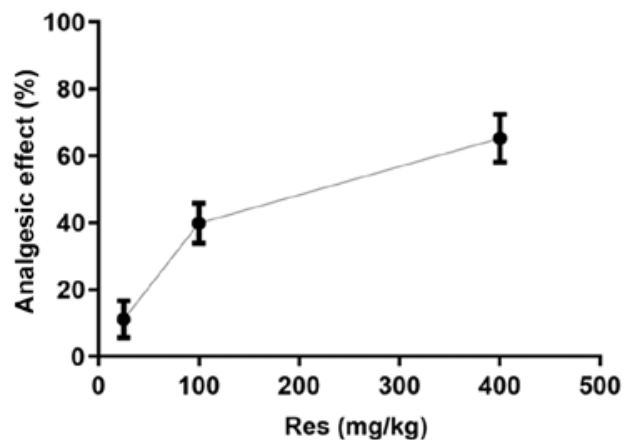

B

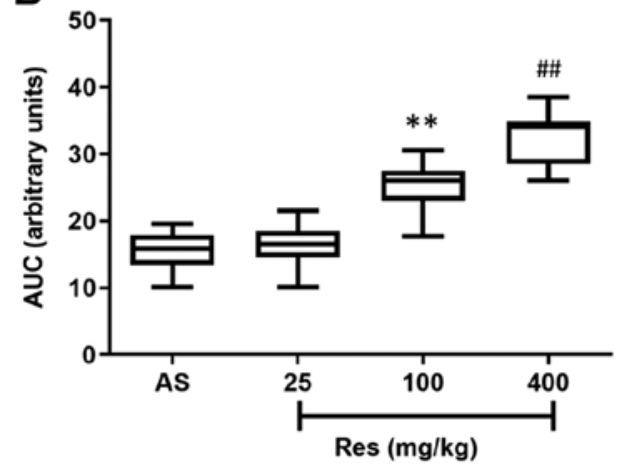

D

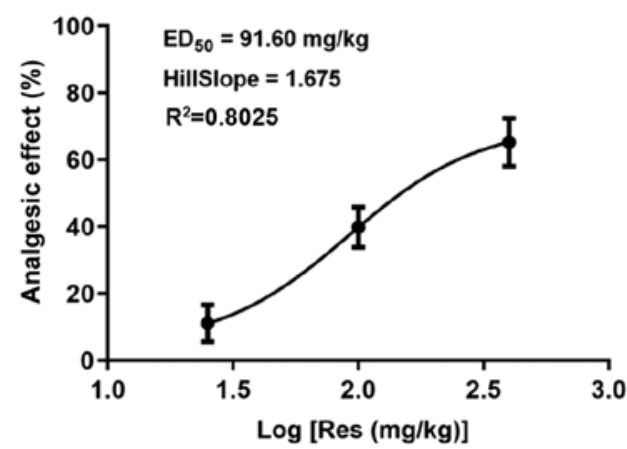

Figure 3. RES inhibits DMA-related behaviors in a dose-dependent manner. (A) Duration of analgesia of various intragastric doses of RES. The results were repeatedly measured 5 times on 1 day pre- and 7, 14, 21 and 28 day post-STZ injections. (B) The AUC of each of the groups. (C) Dose-effect and (D) $\log$ (dose)-effect curves for the analgesic effects of DMA following intragastric RES/AS administration. ${ }^{* *} \mathrm{P}<0.01$ vs. DMA + AS; ${ }^{\# \#} \mathrm{P}<0.01$ vs. DMA + AS RES, resveratrol; DMA, diabetic mechanical allodynia; AUC, area under the time-course curve; AS, alcohol-saline; i.g. intragastric.

ANOVA followed by Bonferroni's post hoc test, respectively. Statistical analyses were performed using GraphPad Prism software (version 5.01; GraphPad Software, Inc.). P<0.05 was considered to indicate a statistically significant difference.

\section{Results}

Characterization of DMA model rats. Rats in the vehicle group maintained normal blood glucose levels, whereas rats in the DM group developed hyperglycemia during the 4 weeks following the STZ injection (day 7: $27.1 \pm 1.3$ vs. $7.35 \pm 0.6 \mathrm{mmol} / 1 ; \mathrm{P}<0.01$; Fig. 2A). Increased body weight was significantly impaired in the DM group compared with the vehicle group (day 7 :
$214.0 \pm 13.0$ vs. $245.0 \pm 11.3$ g; P<0.01; Fig. 2B). Furthermore, rats in the DM group displayed a significant decrease in PWT on day 7 post-STZ injection compared with the vehicle group, which indicated that the DMA had been successfully induced (day $7: 5.7 \pm 1.5$ vs. $11.7 \pm 2.4 \mathrm{~g}$; $\mathrm{P}<0.01$; Fig. $2 \mathrm{C}$ ). Moreover, the DM group rats displayed evident mechanical allodynia from day 7-28 post-STZ injection, with a peak on day 14 compared with the vehicle group (Fig. 2C).

RES alleviates STZ-induced diabetic mechanical allodynia in rats. The analgesic effects of RES on DMA-related behaviors were investigated. Compared with the DMA + AS group, both the DMA + Res $(400 \mathrm{mg} / \mathrm{kg})$ and DMA + Res $(100 \mathrm{mg} / \mathrm{kg})$ 

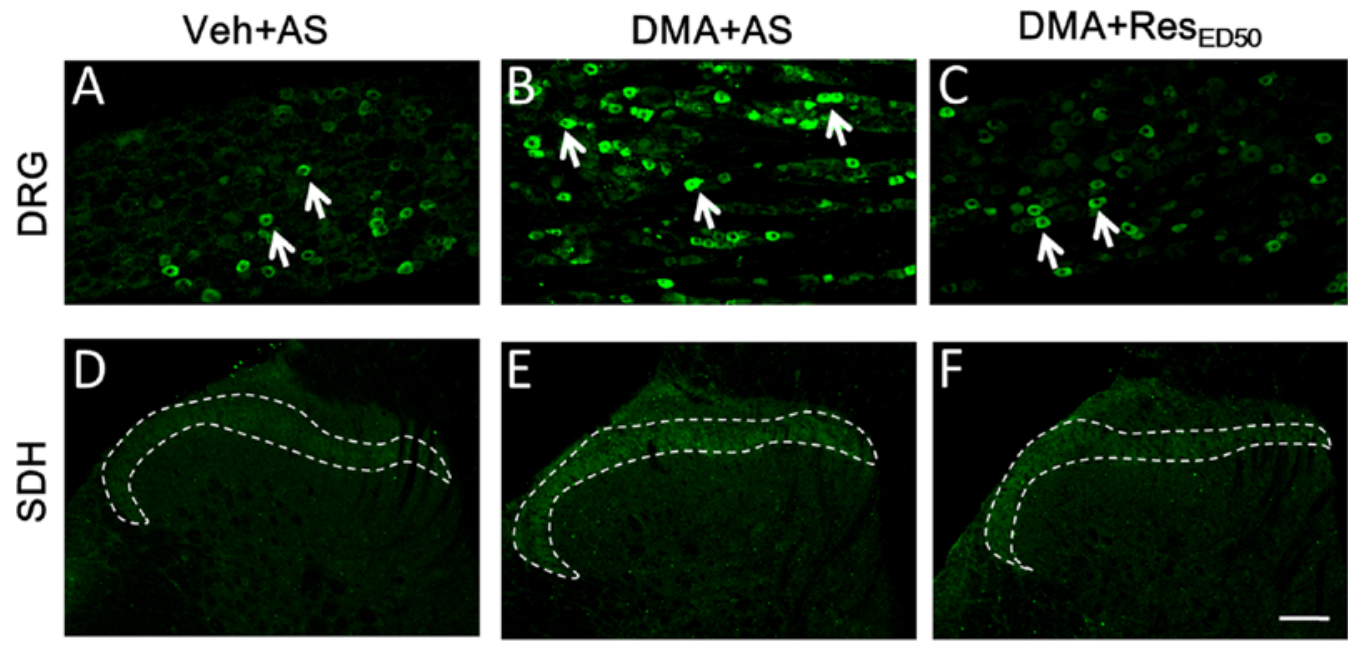

G
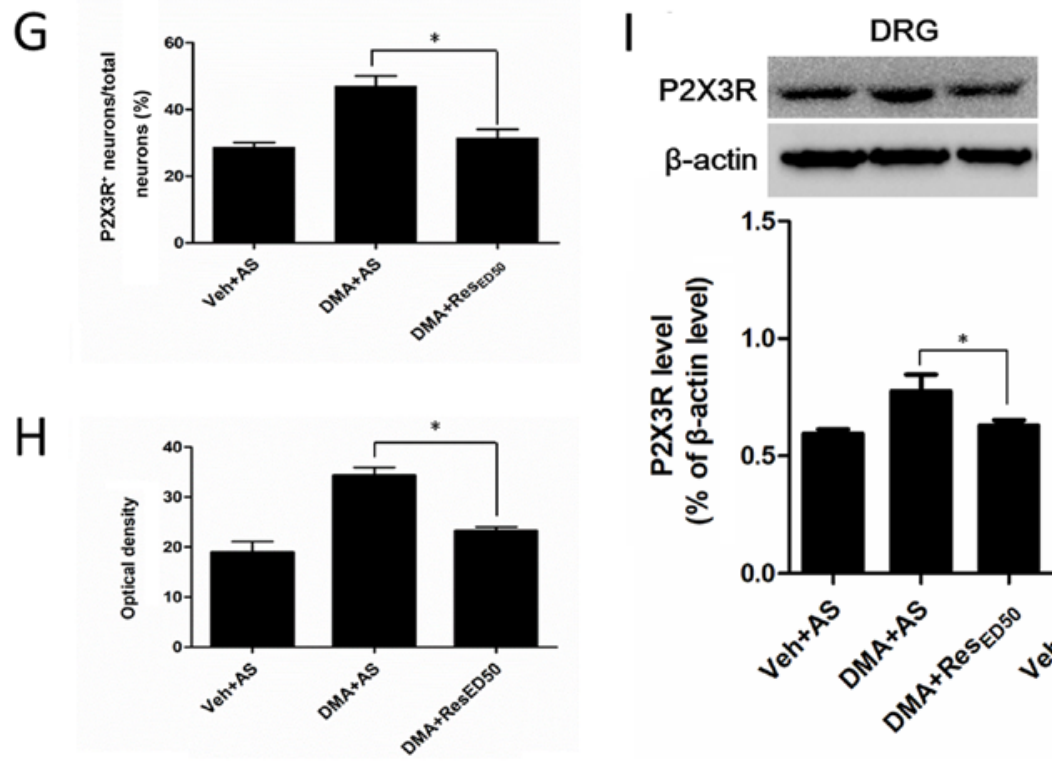

SDH

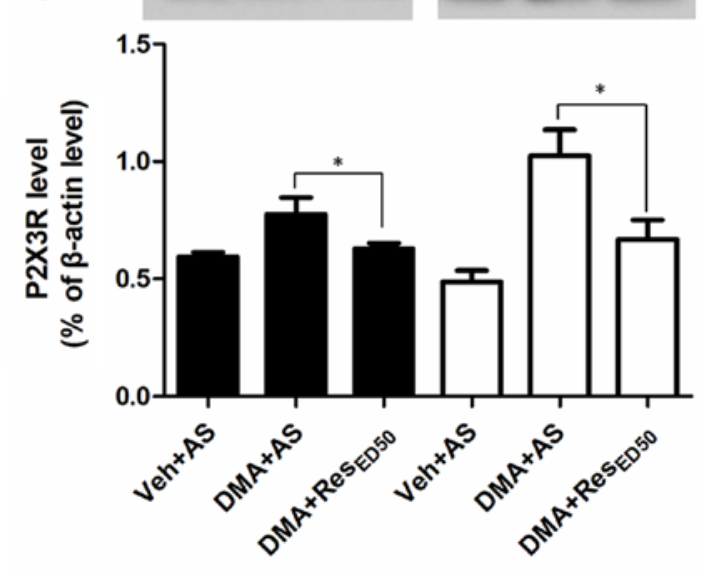

Figure 4. Alterations to P2X3R expression in the DRG and SDH. Representative images of P2X3R staining in the DRG of the (A) Veh + AS, (B) DMA + AS and (C) DMA + RES ${ }_{\mathrm{ED} 50}$ groups. Arrows indicate typical P2X3R-IR (scale bar=100 $\mu \mathrm{m}$ ). Representative images of P2X3R staining in the SDH of the (D) Veh + AS, (E) DMA + AS and (F) DMA + RESED50 groups. Dotted lines indicate typical P2X3R-IR (scale bar=100 $\mu \mathrm{m}$ ). (G) Quantification of the percentage of P2X3R-positive neurons/total neurons in the DRG. (H) Optical density analysis of P2X3R staining in the SDH. (I) P2X3R protein expression levels were measured by western blotting in the DRG and SDH. ${ }^{*} \mathrm{P}<0.05$, as indicated. P2X3R, purinergic receptor P2X3; DRG, dorsal root ganglion; SDH, spinal dorsal horn; Veh, vehicle; AS, alcohol-saline; DMA, diabetic mechanical allodynia; RES, resveratrol; IR, immunoreactivity.

groups significantly increased the PWT, with the maximal pain-relieving effect observed on day 21 post-STZ injection [DMA + Res (400 mg/kg), 9.8 $\pm 1.7 ; \mathrm{DMA}+\operatorname{Res}(100 \mathrm{mg} / \mathrm{kg})$, $7.5 \pm 0.9$; DMA + AS, $1.8 \pm 0.7$; $<<0.01$; Fig. 3A]. After RES administration was terminated on day 22 post-STZ injection, the enhanced PWT was decreased by day 28 post-STZ injection to a level similar to the sensitized level of DMA + AS group; however, the PWT of the DMA + RES (400 mg/kg) group was still significantly increased compared with the DMA + AS group. Subsequently, as indicated by the AUC values of PWT, 100 and $400 \mathrm{mg} / \mathrm{kg}$ RES displayed a significant analgesic effect on DMA compared with the DMA + AS group (Fig. 3B). Furthermore, the effects of various doses of RES on the PWT were assessed using the dose or $\log (\mathrm{dose})$ vs. response curve, and $\mathrm{RES}_{\mathrm{ED} 50}$ was calculated as $91.60 \mathrm{mg} / \mathrm{kg}$ (Fig. 3C and D).

$R E S_{E D 50}$ downregulates $P 2 X 3 R$ expression in DMA model rats. A previous study has indicated that the protein expression of $\mathrm{P} 2 \mathrm{X} 3 \mathrm{R}$ is significantly increased during the development of DMA, particularly on day 21 post-DMA induction (23). Therefore, whether P2X3R was associated with the analgesic effects of RES on DMA-related behaviors was investigated. Compared with the Veh + AS group, the expression of P2X3R was notably increased in the DMA + AS group, particularly in the middle- and small-sized neurons in the DRG (as indicated by arrows in Fig. 4B) and in the fibers of laminae I and II in the SDH (as indicated by dotted lines in Fig. 4E). P2X3R expression was markedly decreased in the DRG and SDH in the DMA + $\mathrm{RES}_{\mathrm{ED} 50}$ group compared with the DMA + AS group (Fig. 4C and F). The percentage of P2X3R-positive neurons/total neurons was significantly decreased in the DMA + $\mathrm{RES}_{\mathrm{ED} 50}$ group compared with the DMA + AS group, decreasing from 47 to $31 \%(\mathrm{P}<0.05$; Fig. 4G). Similar alterations to P2X3R expression levels were observed in the SDH (Fig. 4H), whereby the optical density of $\mathrm{P} 2 \mathrm{X} 3 \mathrm{R}$-immunoreactive fibers in the DMA + $\mathrm{RES}_{\mathrm{ED} 50}$ group 
was significantly lower compared with the DMA + AS group. The results indicated that inhibition of P2X3R overexpression may be the mechanism underlying RES-mediated alleviation of DMA-related behaviors.

To further investigate the aforementioned observations, P2X3R protein expression was examined by western blotting. Similarly, in both the DRG and SDH, P2X3R expression was significantly decreased in DMA tissues following $\mathrm{RES}_{\mathrm{ED} 50}$ treatment compared with the DMA + AS group $(\mathrm{P}<0.05$; Fig. 4I).

\section{Discussion}

In the present study, the effects and possible mechanisms underlying RES in STZ-induced DMA model rats were assessed using behavioral pharmacological methods, as well as morphological and molecular biological analyses. The results indicated that: i) The intragastric administration of RES effectively alleviated DMA-associated pain behaviors; ii) the $\mathrm{ED}_{50}$ of RES was $91.6 \mathrm{mg} / \mathrm{kg}$ for analgesic effects on DMA-related behaviors; and iii) in the DRG neurons and SDH terminals, the protein expression level of P2X3R was significantly decreased after $\operatorname{RES}_{\mathrm{ED} 50}$ administration for 14 continuous days. The results suggested that RES effectively relieved STZ-induced DMA in rats and that downregulation of P2X3R expression may be a possible mechanism underlying RES-mediated effects.

RES displays anti-inflammatory, antioxidative, antitumorigenic and neuroprotective effects (32-34). It has also been reported that RES attenuates mechanical allodynia and thermal hyperalgesia in rats with chronic constriction injury by enhancing interleukin (IL)-4 receptor-mediated anti-inflammatory responses in the spinal cord (35). A previous study demonstrated that mechanical allodynia was present within the 2 weeks following STZ-induced diabetes and persisted for a further 7 weeks (36). It has also been reported that the daily oral administration of RES from week 4 post-STZ injection mitigates thermal hyperalgesia in diabetic model mice (16). In the present study, the intragastric administration of RES effectively alleviated DMA-associated pain behaviors in a dose-dependent manner from week 2-4 post-STZ injection. Collectively, the results suggested that RES displayed an analgesic effect against mechanical allodynia in DM model rats.

DNP-associated hyperglycemia can lead to DRG dysfunction, and may affect some ion channels or receptors in neurons (37). Transient receptor potential vanilloid 1 and voltage-gated sodium channel $\mathrm{Na}_{\mathrm{V}} 1.7$ expression are significantly increased in DRG neurons of DNP model rats $(31,38)$. It has been reported that increased P2X3R or P2X7R expression in the DRG in an animal model of neuropathic pain is induced by pSNL or chronic constriction injury, respectively. RES treatment decreases the expression of P2X3R or P2X7R, which indicates that RES alleviates pain behavior in rats with neuropathic pain by inhibiting the upregulated expression of $\mathrm{P} 2 \mathrm{X}$ receptors $(11,22)$. In a previous study, P2X3R protein expression levels increased in the DRG and SDH with the development of DMA (23). In the present study, the results suggested that repetitive administration of RES downregulated P2X3R overexpression in DRG neurons and SDH terminals in DMA model rats. The results indicated that RES may ameliorate DMA by attenuating increased P2X3R expression in DMA model rats.

However, only the preliminary mechanism underlying RES-mediated alleviation of STZ-induced diabetic mechanical allodynia was investigated in the present study. According to a previous study, $\mathrm{P} 2 \mathrm{X} 3$ receptor can participate in mechanical and thermal pain in pSNL model rats via the ERK signaling pathway (22). Some proinflammatory cytokines, such as tumor necrosis factor- $\alpha$ and IL-1 $\beta$, also activate signaling pathways in peripheral sensory neurons, leading to downstream activation/sensitization of P2X3 (39). In addition, RES can inhibit microglia activation via the AMP-activated protein kinase signaling pathway, thereby reducing morphine tolerance (40). Therefore, future studies should investigate the association between RES and the ERK signaling pathways and proinflammatory cytokines.

In conclusion, the present study suggested that RES displayed an analgesic effect against STZ-induced DMA and the underlying mechanism may involve downregulation of P2X3R expression. Therefore, RES may serve as a potential therapeutic target for DMA.

\section{Acknowledgements}

Not applicable.

\section{Funding}

The present study was supported by the National Natural Science Foundation of China (grant nos. 31600951 and 81873740), the Natural Science Basic Research Plan in Shaanxi Province of China (grant nos. 2017JQ8048, 2018JM7094 and 2018JQ8003), the Research Plan of Xi'an Medical University (grant nos.2017PT28 and 2018PT11) and The Youth Innovation Team of Shaanxi University.

\section{Availability of data and materials}

The datasets used and/or analyzed during the current study are available from the corresponding author on reasonable request.

\section{Authors' contributions}

YC, LL and XG conceived and designed the study. YC, YL and JN performed the majority of the experiments. LL, YM, $\mathrm{XW}$ and $\mathrm{ZQ}$ analyzed the data and conducted some of the experiments. YC and XG contributed reagents, materials and analysis tools. YC and LL drafted the manuscript. XCG revised the manuscript. All authors read and approved the final manuscript.

\section{Ethics approval and consent to participate}

The present study was approved by the Animal Care and Ethical Committee at Xi'an Medical University.

\section{Patient consent for publication}

Not applicable. 


\section{Competing interests}

The authors declare that they have no competing interests.

\section{References}

1. American Diabetes Association: (2) Classification and diagnosis of diabetes. Diabetes Care 38 (Suppl): S8-S16, 2015.

2. Guastella V and Mick G: Strategies for the diagnosis and treatment of neuropathic pain secondary to diabetic peripheral sensory polyneuropathy. Diabetes Metab 35: 12-19, 2009.

3. Rajchgot T, Thomas SC, Wang JC, Ahmadi M, Balood M, Crosson T, Dias JP, Couture R, Claing A and Talbot S: Neurons and Microglia; A sickly-sweet duo in diabetic pain neuropathy. Front Neurosci 13: 25, 2019.

4. Scholz J, Rathmell JP, David WS, Chad DA, Broderick AC, Perros SG, Shin NS, Wells JL, Davis JB, DiMaggio CJ, et al: A standardized clinical evaluation of phenotypic diversity in diabetic polyneuropathy. Pain 157: 2297-2308, 2016.

5. Dubin AE and Patapoutian A: Nociceptors: The sensors of the pain pathway. J Clin Invest 120: 3760-3772, 2010.

6. Castro OW, Upadhya D, Kodali M and Shetty AK: Resveratrol for easing status epilepticus induced brain injury, inflammation, epileptogenesis, and cognitive and memory dysfunction-are we there yet? Front Neurol 8: 603, 2017.

7. Daverey A and Agrawal SK: Pre and post treatment with curcumin and resveratrol protects astrocytes after oxidative stress. Brain Res 1692: 45-55, 2018.

8. Lancon A, Frazzi R and Latruffe N: Anti-oxidant, anti-inflammatory and anti-angiogenic properties of resveratrol in ocular diseases. Molecules 21: 304, 2016.

9. Ding Z, Cao J, Shen Y, Zou Y, Yang X, Zhou W, Guo Q and Huang C: Resveratrol promotes nerve regeneration via activation of p300 acetyltransferase-mediated VEGF signaling in a rat model of sciatic nerve crush injury. Front Neurosci 12: 341, 2018

10. Oda H, Ohta S, Ikeguchi R, Noguchi T, Kaizawa Y, Yurie H, Takeuchi H, Mitsuzawa S and Matsuda S: Pretreatment of nerve grafts with resveratrol improves axonal regeneration following replantation surgery for nerve root avulsion injury in rats. Restor Neurol Neurosci 36: 647-658, 2018.

11. Xie J, Liu S, Wu B, Li G, Rao S, Zou L, Yi Z, Zhang C, Jia T, Zhao S, et al: The protective effect of resveratrol in the transmission of neuropathic pain mediated by the $\mathrm{P} 2 \mathrm{X}_{7}$ receptor in the dorsal root ganglia. Neurochem Int 103: 24-35, 2017.

12. Zou P, Liu X, Li G and Wang Y: Resveratrol pretreatment attenuates traumatic brain injury in rats by suppressing NLRP3 inflammasome activation via SIRT1. Mol Med Rep 17: 3212-3217, 2018 .

13. Zhao H, Chen S, Gao K, Zhou Z, Wang C, Shen Z, Guo Y, Li Z, Wan Z, Liu C and Mei X: Resveratrol protects against spinal cord injury by activating autophagy and inhibiting apoptosis mediated by the SIRT1/AMPK signaling pathway. Neuroscience 348: 241-251, 2017.

14. Tao L, Ding Q, Gao C and Sun X: Resveratrol attenuates neuropathic pain through balancing pro-inflammatory and anti-inflammatory cytokines release in mice. Int Immunopharmacol 34: 165-172, 2016.

15. Yang YJ, Hu L, Xia YP, Jiang CY, Miao C, Yang CQ, Yuan M and Wang L: Resveratrol suppresses glial activation and alleviates trigeminal neuralgia via activation of AMPK. J Neuroinflammation 13: 84, 2016.

16. Sharma S, Kulkarni SK and Chopra K: Effect of resveratrol, a polyphenolic phytoalexin, on thermal hyperalgesia in a mouse model of diabetic neuropathic pain. Fundam Clin Pharmacol 21: 89-94, 2007.

17. Sharma S, Chopra K and Kulkarni SK: Effect of insulin and its combination with resveratrol or curcumin in attenuation of diabetic neuropathic pain: participation of nitric oxide and TNF-alpha. Phytother Res 21: 278-283, 2007.

18. Sharma SS, Kumar A, Arora M and Kaundal RK: Neuroprotective potential of combination of resveratrol and 4-amino 1,8 naphthalimide in experimental diabetic neuropathy: Focus on functional, sensorimotor and biochemical changes. Free Radic Res 43: 400-408, 2009.

19. Edwards FA and Gibb AJ: ATP-a fast neurotransmitter. FEBS Lett 325: 86-89, 1993.

20. Bernier LP, Ase AR and Séguéla P: P2X receptor channels in chronic pain pathways. Br J Pharmacol 175: 2219-2230, 2018.
21. Zhang HH, Hu J, Zhou YL, Qin X, Song ZY, Yang PP, Hu S, Jiang $X$ and $\mathrm{Xu} G Y$ : Promoted interaction of nuclear factor $-\kappa \mathrm{B}$ with demethylated purinergic $\mathrm{P} 2 \mathrm{X} 3$ receptor gene contributes to neuropathic pain in rats with diabetes. Diabetes 64: 4272-4284, 2015.

22. Guo J, Wang C, Niu X, Zhou F, Li H and Gao W: Effects of resveratrol in the signaling of neuropathic pain involving $\mathrm{P} 2 \mathrm{X} 3$ in the dorsal root ganglion of rats. Acta Neurol Belg: Apr 19, 2019 (Epub ahead of print).

23. Cui YY, Wu HH, Wa L, Shi J and Li YQ: Spatio-temporal expression of $\mathrm{P} 2 \mathrm{X} 3$ receptor in rats with diabetic mechanical allodynia. Acta Anatomica Sinica 45: 540-544, 2014.

24. Zimmermann M: Ethical guidelines for investigations of experimental pain in conscious animals. Pain 16: 109-110, 1983.

25. Chaplan SR, Bach FW, Pogrel JW, Chung JM and Yaksh TL: Quantitative assessment of tactile allodynia in the rat paw. J Neurosci Methods 53: 55-63, 1994.

26. Wu HH, Yin JB, Zhang T, Cui YY, Dong YL, Chen GZ and Wang W: Inhibiting spinal neuron-astrocytic activation correlates with synergistic analgesia of dexmedetomidine and ropivacaine. PLoS One 9: e92374, 2014.

27. Yin JB, Zhou KC, Wu HH, Hu W, Ding T, Zhang T, Wang LY, Kou JP, Kaye AD and Wang W: Analgesic effects of Danggui-Shaoyao-San on various 'Phenotypes' of nociception and inflammation in a formalin pain model. Mol Neurobiol 53: 6835-6848, 2016.

28. Tallarida RJ: Drug synergism: Its detection and applications. J Pharmacol Exp Ther 298: 865-872, 2001.

29. Ke HH, Wu HH, Zhu Q, Zhang Y, Xiao JR, Su XZ, Zheng WJ, Cai YP, Wu XZ, Wang YT and Chen GZ: Neuromuscular effect of dexmedetomidine on sevoflurane: An open-label, dose-escalation clinical trial. Minerva Anestesiol 83: 790-797, 2017.

30. Bai L, Wang W, Dong YL, Wang W, Huang J, Wang XY, Wang LY, Li YQ and Wu SX: Attenuation of mouse somatic and emotional inflammatory pain by hydralazine through scavenging acrolein and inhibiting neuronal activation. Pain Physician 15: 311-326, 2012.

31. Cui YY, Xu H, Wu HH, Qi J, Shi J and Li YQ: Spatio-temporal expression and functional involvement of transient receptor potential vanilloid 1 in diabetic mechanical allodynia in rats. PLoS One 9: e102052, 2014.

32. Baur JA and Sinclair DA: Therapeutic potential of resveratrol: The in vivo evidence. Nat Rev Drug Discov 5: 493-506, 2006.

33. Shimazu Y, Shibuya E, Takehana S, Sekiguchi K, Oshima K, Kamata $\mathrm{H}$, Karibe $\mathrm{H}$ and Takeda M: Local administration of resveratrol inhibits excitability of nociceptive wide-dynamic range neurons in rat trigeminal spinal nucleus caudalis. Brain Res Bull 124: 262-268, 2016.

34. Takehana S, Sekiguchi K, Inoue M, Kubota Y, Ito Y, Yui K, Shimazu Y and Takeda M: Systemic administration of resveratrol suppress the nociceptive neuronal activity of spinal trigeminal nucleus caudalis in rats. Brain Res Bull 120: 117-122, 2016.

35. Xu M, Cheng Z, Ding Z, Wang Y, Guo Q and Huang C: Resveratrol enhances IL-4 receptor-mediated anti-inflammatory effects in spinal cord and attenuates neuropathic pain following sciatic nerve injury. Mol Pain 14: 1744806918767549, 2018.

36. Zou L, Gong Y, Liu S and Liang S: Natural compounds acting at $\mathrm{P} 2$ receptors alleviate peripheral neuropathy. Brain Res Bull 151: 125-131, 2019.

37. Schreiber AK, Nones CF, Reis RC, Chichorro JG and Cunha JM: Diabetic neuropathic pain: Physiopathology and treatment. World J Diabetes 6: 432-444, 2015.

38. McDonnell A, Collins S, Ali Z, Iavarone L, Surujbally R, Kirby S and Butt RP: Efficacy of the Nav1.7 blocker PF-05089771 in a randomised, placebo-controlled, double-blind clinical study in subjects with painful diabetic peripheral neuropathy. Pain 159: 1465-1476, 2018.

39. Schiavuzzo JG, Teixeira JM, Melo B, da Silva dos Santos DF, Jorge CO, Oliveira-Fusaro MC and Parada CA: Muscle hyperalgesia induced by peripheral $\mathrm{P} 2 \mathrm{X} 3$ receptors is modulated by inflammatory mediators. Neuroscience 285: 24-33, 2015.

40. Han Y, Jiang C, Tang J, Wang C, Wu P, Zhang G, Liu W, Jamangulova N, Wu X, and Song X: Resveratrol reduces morphine tolerance by inhibiting microglial activation via AMPK signalling. Eur J Pain 18: 1458-1470, 2014.

his work is licensed under a Creative Commons Attribution-NonCommercial-NoDerivatives 4.0 International (CC BY-NC-ND 4.0) License. 